\title{
Les enjeux philosophiques laïques de la bioéthique
}

\author{
Gilbert Hottois
}

\section{(2) OpenEdition}

Journals

Édition électronique

URL : http://journals.openedition.org/ries/4151

DOI : 10.4000/ries.4151

ISSN : 2261-4265

\section{Éditeur}

Centre international d'études pédagogiques

\section{Édition imprimée}

Date de publication : 5 mars 1995

Pagination : $43-53$

ISSN : 1254-4590

\section{Référence électronique}

Gilbert Hottois, "Les enjeux philosophiques laïques de la bioéthique », Revue internationale d'éducation de Sèvres [En ligne], 05 | 1995, mis en ligne le 16 mars 2015, consulté le 30 avril 2019. URL : http:// journals.openedition.org/ries/4151; DOI : 10.4000/ries.4151

Ce document a été généré automatiquement le 30 avril 2019

(c) Tous droits réservés 


\title{
Les enjeux philosophiques laïques de la bioéthique
}

\author{
Gilbert Hottois
}

1 La bioéthique est certainement l'une des expressions majeures de l'affairement éthique contemporain. Elle constitue en outre une excellente illustration de la manière dont la question éthique se pose aujourd'hui dans les sociétés développées complexes modernes, caractérisées par le multiculturalisme et la dynamique technoscientifique. Bien comprise, la bioéthique ne fait pas apparaître l'éthique sous un jour simple; elle manifeste, au contraire, toutes les implications économiques et politiques de questions morales apparemment les plus "privées». L'expérience m'a enseigné qu'une part très considérable de ce que l'on appelle la bioéthique serait plus adéquatement nommée «biopolitique » ou « bioéconomie ».

\section{Qu'est-ce que la bioéthique?}

Le mot bioethics est forgé il y a presque vingt-cinq ans par un biologiste américain, Van Rensselaer Potter, qui l'utilise dans le titre d'un article: "Bioethics, the science of survival » (1970); il le reprend l'année suivante dans le titre de son livre: Bioethics, Bridge to the Future (Englewood Cliffs, 1971). Potter définit la bioéthique comme la discipline normative du bien-vivre et du mieux-vivre prenant directement appui sur les progrès des sciences biologiques et médicales fortement valorisées. Potter croit aux sciences et aux techniques, à l'éducation et à la culture technoscientifiques pour améliorer la condition humaine. Si j'y insiste, c'est parce que l'évolution ultérieure de la bioéthique, n'a, le plus souvent, pas préservé cet optimisme progressiste et cette confiance dans le rationalisme 
scientifique. Au contraire, très tôt, la bioéthique s'est mise à encourager des réactions et des phantasmes techno- et sciento-phobes.

D’une manière générale, le développement de la bioéthique est à comprendre sur fond d'une double crise ou mutation:

- la crise des valeurs et des normes collectives stables et unifiantes qui va de pair avec le développement moderne de l'individualisme des personnes et du pluralisme des sociétés; dans une société moralement unitaire et stable, il n'y aurait pas de débat bioéthique ;

- la crise ou mutation de la médecine traditionnelle naturaliste et paternaliste qui va de pair avec le développement de la médecine expérimentale moderne et des techno-sciences biomédicales aujourd'hui. Celles-ci ont contribué à une naturalisation de l'humain (l'homme n'est qu'un vivant parmi les vivants) et à son « opérationnalisation » : le vivant (y compris l'homme) est un complexe biophysique, produit contingent de l'évolution, et, par conséquent, modifiable, manipulable, reconstructible, opérable, dans tous ses aspects et diversement.

4 Si vous rapprochez ces deux crises ou mutations (celle des valeurs avec développement de l'individualisme et du pluralisme, et celle de la médecine de plus en plus technicienne et puissante), vous vous retrouvez au cœur du problème bioéthique : dans quelle mesure l'individu peut-il disposer de la réalité biophysique (son corps) qu'il est selon sa liberté et son désir? Dans quelle mesure les autres (la société, le droit, l'État) peuvent-ils restreindre ou contraindre cette liberté ? Et que deviennent la conscience et la liberté médicales dans ce contexte? Il est facile d'illustrer ces interrogations : songeons à des questions aussi banales que la contraception ou l'avortement, ou à des questions plus neuves telles que la procréatique et les familles monoparentales ou homosexuelles, ou le diagnostic génétique, ou le droit à l'euthanasie, ou la médecine des prothèses, de convenance ou de confort, ou encore les transferts d'organes...

5 Comme dans tant d'autres domaines, il y a, au départ éloigné de la bioéthique, la seconde guerre mondiale qui a cristallisé d'abord les phantasmes antiscience et anti-technique autour de l'atome. Mais beaucoup plus spécifiquement, la référence quasi fondatrice de la nouvelle éthique est l'expérimentation réalisée sur des personnes non consentantes par des médecins nazis. La condamnation de ceux-ci en 1947 par le tribunal militaire américain de Nuremberg fut l'occasion d'un texte, connu sous le nom de «Code de Nuremberg", qui définit strictement les conditions auxquelles l'expérimentation sur l'homme est moralement admissible. Trois principes sont ainsi exprimés :

- le consentement informé et volontaire du sujet de l'expérience ;

- la balance positive des risques (pour le sujet) et des bienfaits (pour le sujet et/ou pour la société) : finalité humanitaire de l'expérience et réversibilité des dommages éventuels;

- la scientificité de la méthode et la compétence de l'expérimentateur.

Le Code de Nuremberg réagit donc contre les tentations de la recherche technoscientifique conduisant à l'objectivation et à l'instrumentalisation abusives des sujets, et au mépris de leur liberté. Cette liberté doit demeurer inaliénable pour toute décision les concernant et le médecin a le devoir d'éclairer le choix sans se substituer au patient qui choisit. Le Code de Nuremberg est contemporain de la Déclaration universelle des droits de l'homme (1948), dont il partage l'esprit de sauvegarde de la dignité des personnes et des libertés individuelles contre les abus des pouvoirs institués. Le Code et la Déclaration demeureront des sources d'inspiration constantes pour tous les grands textes de bioéthique et d'éthique médicale de la seconde moitié de ce siècle, telle que 
l'importante déclaration d'Helsinki publiée primitivement en 1964 par l'Association médicale mondiale.

Dès les années cinquante, des théologiens, notamment J. Fletcher (américain et protestant), réfléchissent sur l'éthique médicale du point de vue du patient dont est souligné le droit à l'information et à l'autonomie (Morals and medecine, 1954).

$\mathrm{Au}$ cours des années soixante et soixante-dix se multiplient les dénonciations et les condamnations de l'expérimentation sur l'homme, soit que l'expérimentation ait été insuffisante (l'affaire de la thalidomide), soit, le plus souvent, qu'elle ait été menée à l'insu des cobayes choisis parmi des groupes sociaux vulnérables ou marginaux (injection de cellules cancéreuses à des patients séniles, du virus hépatique à des enfants retardés, expériences sur des Noirs, des prisonniers, etc.).

9 Très vite une synergie opère entre le versant « éthique médicale » de la bioéthique et le versant "éco-éthique ou éthique environnementale ", puisque au cours de la même période, on rencontre les premières grandes alertes à la pollution (l'affaire du Torrey Canyon en 1967) et les inquiétudes à propos de la dissémination dans le milieu d'organismes génétiquement manipulés, entraînant les généticiens à respecter, entre 1974 et 1975, un moratoire (dit "d'Asilomar ») sur les manipulations génétiques des micro-organismes.

10 À partir de cette époque, les faits «bio-éthiquement significatifs » deviennent à ce point nombreux, que je me limite à une énumération très sommaire et pas du tout exhaustive. Voici quelques repères parmi les plus importants.

11 Dès le milieu des années soixante sont créés aux États-Unis les Institutional Review Boards (IRB): ce sont des comités d'évaluation scientifique et éthique de la recherche. Aujourd'hui, de tels comités d'éthique de la recherche existent partout où se déroule la recherche biomédicale. Ce principe d'une surveillance éthique et collective de la recherche est nouveau et capital.

12 Autour de 1970, sont fondés les deux grands centres de recherches qui demeurent aujourd'hui encore des pôles essentiels pour la bioéthique mondiale : le Hastings Center (près de New York) et le Kennedy Institute of Ethics (près de Washington); en 1978 paraissent les quatre volumes de l'Encyclopedia of Bioethics, qui consacre l'existence de la nouvelle discipline.

13 L'institutionnalisation de la réflexion éthique collective, pluridisciplinaire et pluraliste sur la recherche et développement technoscientifique (RDTS) biomédicale va s'étendre et faire apparaître de plus en plus de dimensions à portée politique nationale, internationale, voire mondiale. Ainsi vont se développer les Comités d'éthique à l'échelon national et multinational :

- en 1974, le Congrès américain crée la National Commission for the Protection of Human Subjects and Behavioral Research qui débouche sur le fameux Belmont Report; d'autres commissions nationales de ce type ad hoc suivront aux États-Unis (les President's Commissions) et ailleurs (la célèbre commission Warnock en Grande-Bretagne) ;

- d'autres pays vont instituer des commissions permanentes non limitées à une problématique déterminée, telle la France qui crée en 1983 son comité consultatif national d'éthique pour les sciences de la vie et de la santé ; cet exemple sera suivi par un nombre croissant de pays, dont récemment la Belgique ;

- au plan multinational, signalons la création en 1985, par le Conseil de l'Europe, d'une instance qui s'appelle aujourd'hui le comité directeur pour la bioéthique et qui vient de 
publier une convention pour la bioéthique; la mise sur pied en 1992 d'un "groupe de conseillers pour l'éthique de la biotechnologie » auprès de la présidence de la Commission européenne ; la création en 1993 du comité international de bioéthique de l'UNESCO.

Même sommaire, l'énumération est imposante et manifeste l'extraordinaire affairement éthico-politique autour de la RDTS biomédicale. Car il faut ajouter à cela des milliers de comités d'éthique clinique et/ou de la recherche formés dans les hôpitaux et les facultés, ainsi que les centaines de centres de bioéthique qui ne cessent de fleurir à travers le monde.

\section{Les enjeux}

S'il fallait répondre en quelques mots, je dirais que l'enjeu est la sauvegarde et l'extension à tous les individus d'une liberté collective.

\section{Attaques contre la modernité}

Le champ de la bioéthique est envahi par une foule de personnalités qui chargent la modernité de tous les maux et qui, surtout, prétendent connaître les solutions qui sauvent, généralement puisées dans les « vraies » valeurs de l'une ou l'autre tradition que les anti-valeurs modernes auraient bafouées.

Plus précisément, deux boucs émissaires sont chargés de tous les maux :

- la science moderne, et plus encore la technique ou la techno-science contemporaine ;

- l'individualisme interprété comme égoïsme, le pluralisme compris comme relativisme sceptique et indifférence généralisée, la tolérance ramenée au laxisme.

Cette double dénonciation, qu'il n'est d'ailleurs pas exceptionnel de rencontrer aussi chez des interlocuteurs qui se définiraient comme laïques, concerne, en fait, la dynamique qui s'est mise en route à partir des $\mathrm{XVII}^{\mathrm{e}}$ et XVIII ${ }^{\mathrm{e}}$ siècles en Europe, une dynamique d'émancipation et de progrès illimités, qui s'appuie amplement sur le développement des sciences et des techniques, ainsi que sur l'extension à tous les hommes, grâce à l'éducation et à l'enseignement d'une culture universelle basée sur la philosophie des droits de l'homme. C'est pourquoi la vigilance devrait porter prioritairement sur la sauvegarde de la liberté de la recherche scientifique et sur celle des libertés individuelles.

\section{La rhétorique bioéthique et ses dangers}

19 Cette défense de la liberté n'est possible que si l'on ne nie pas les problèmes, les risques et les inconnues associés à la modernité. Il me paraît capital de faire la part entre les dangers réels et les dangers symboliques ou imaginaires. La bioéthique est malheureusement envahie par les amalgames, les confusions, les extrapolations, les exagérations qui exploitent la fascination trouble et l'angoisse vague de l'inconnu ou du mal connu, et entourent les entreprises et réalisations technoscientifiques d'un halo de promesses et de périls fictifs. Cela concerne déjà les mots et les images utilisées pour les illustrer. Ainsi ne cesse-t-on de parler de «bébé éprouvette », alors qu'il ne s'agit jamais de plus de huit, seize ou trente-deux cellules; le "clonage » évoque la multiplication identique d'un même individu, alors que l'expérience tentée concernait la division d'un embryon de quelques cellules et qui ne pouvait se développer car il était anormal; le 
terme de "manipulations " génétiques évoque une activité ludique irresponsable, alors que les difficiles recherches portent essentiellement sur des modifications thérapeutiquement ou économiquement utiles; la génétique ou l'entreprise de séquençage du génome humain fait se lever le spectre de l'eugénisme, amalgamé à celui du nazisme, auquel l'on associe aussi volontiers l'euthanasie.

Mais le problème est général : une importante partie des mots de la bioéthique ont une portée rhétorique non négligeable et d'autant plus pernicieuse que la très large majorité des citoyens, même ceux qui sont cultivés et comptent parmi les intellectuels et les universitaires, ne disposent que de ces mots et n'ont nul accès aux choses et aux actes qui sont derrière. Qu'évoquent, dans l'esprit de chacun d'entre nous, des expressions telles que : acharnement thérapeutique, génome humain, banque de sperme ou d'embryon, cobaye humain, mort cérébrale, mère porteuse, droits des animaux, droits des générations futures, expérimentation sur l'embryon, fivete, personne potentielle, transgénose, etc.? Il n'est pas rare de voir des intellectuels, moralistes, philosophes, théologiens, mais aussi des techniciens et des scientifiques étrangers aux sciences biomédicales, s'inquiéter des modifications de l'espèce, en prenant ce mot avec la signification d'essence ou nature humaine, que pourraient entraîner les entreprises de la génétique, sans que ces critiques aient la moindre notion de ces entreprises, et qu'ils mesurent encore moins ce que c'est que le polymorphisme humain et à quel point est grande l'insignifiance de toute intervention génétique effectuée sur des individus même nombreux eu égard à la génétique des populations.

21 Le problème, et c'est un point que beaucoup de scientifiques ont de la peine à comprendre, c'est que des dangers phantasmes et qui ne correspondent à aucun risque physique réel, ont néanmoins leur réalité: celle d'une croyance ou d'une illusion collective dont la portée sociale et politique peut être énorme, et conduire à arrêter des projets de recherche éventuellement prometteurs. L'information claire et appropriée de l'opinion publique et l'acculturation scientifico-technique sérieuse de la jeunesse grâce à un enseignement de qualité me paraissent d'une importance vitale pour l'avenir de la RDTS et donc de notre civilisation dans la mesure où celle-là constitue un moteur de celle-ci.

\section{La liberté de la recherche}

La question de la liberté de la recherche technoscientifique et des modalités de sa régulation me paraît un des centres du débat bioéthique et, comme je l'ai souligné, un des principaux enjeux idéologiques. Cette question est cependant complexe et délicate pour deux raisons principales : l'imprévisibilité et la dépendance.

La dépendance : dans notre société, la recherche fondamentale désintéressée, c'est-à-dire dépourvue de finalités pratiques, a quasiment disparu. La science n'est plus « pure », aussi ne peut-elle se décharger sur les techniques, assimilées à des savoirs appliqués, des responsabilités du bon ou mauvais usage. Tout savoir moderne est opératoire, c'est-à-dire indissociable d'une série de pouvoir-faire techniques. "Savoir égale pouvoir ", avait déjà déclaré au XVIIe siècle, l'un des fondateurs de la science moderne, Francis Bacon. Voilà pourquoi je préfère, personnellement, parler des «techno-sciences ». Or, dans la mesure où le développement du savoir coïncide avec celui du pouvoir, du pouvoir-faire - mais aussi : pouvoir contrôler, maîtriser, prédire, produire, créer, construire (et, bien sûr, détruire) -, il intéresse le politique et l'économie. Il n'y a pas, et cela est particulièrement 
vrai des techno-sciences biomédicales, de domaine de la RDTS qui ne soit investi d'enjeux économiques et politiques importants. Si vous prenez, par exemple, le Projet génome humain, qui est un projet de recherche fondamentale, ses implications et conséquences pratiques et non prévisibles sont incalculables, d'où la bataille juridique (et politique) déjà engagée à propos de ce qui est brevetable ou non. Il est devenu impossible d'abstraire les techno-sciences des aspects économiques et politiques qui ont tendance à motiver leur développement ou, en tous cas, à le conditionner. Les considérations d'opportunité politique, intérieure et extérieure, les questions de rentabilité et de profit, de compétitivité et de concurrence, sont devenues cruciales.

Ceci me conduit à souligner deux choses: la première est que ni la technique ni le libéralisme économique ne sont nécessairement contraires à la liberté de recherche qui se porte mieux dans le monde du marché et du capital qu'ailleurs; la seconde est qu'il ne faut pas imputer aux scientifiques, à la science et à la technique, les abus, les détournements, les excès, l'irresponsabilité, associés en réalité aux inévitables implications économiques et politiques de la RDTS, aux volontés de pouvoir, au désir de profit, à l'argent, tentations auxquelles, bien entendu, les scientifiques aussi peuvent être sensibles. Les causes s'enracinent dans la nature humaine, et dans les défaillances de son éducation.

Le second aspect que je voulais évoquer au sujet de la liberté de la recherche est l'imprévisibilité. Toute recherche authentique est imprévisible, spécialement en ce qui concerne ses retombées à moyen et à long terme; si l'on connaissait à l'avance les résultats d'une expérience, on en ferait l'économie. Toute recherche expérimentale comporte de l'inconnu et du risque. Vouloir contrôler et évacuer totalement celui-ci revient à vouloir arrêter la recherche. Il y a comme un paradoxe entre l'apport de la science une fois qu'elle est constituée et son mode d'acquisition. La science permet de prédire, de contrôler, de maîtriser, d'éliminer les risques. Son acquisition, la recherche, implique le risque, l'avancée aveugle dans l'inconnu. Or, parce qu'elle est scientifique, notre société admet de moins en moins que l'on courre des risques, que l'on ne puisse contrôler, et que l'on ne sache, en cas de malheur, identifier la faute ou l'erreur, et assigner les responsabilités. Le débat bioéthique agite volontiers risques, dangers, les gonfle dans un sens d'autant plus apocalyptique qu'ils restent imprécis et qu'ils semblent devoir affecter l'existence et l'image traditionnelle de l'homme.

Face à ces périls allégués, des tendances puissantes se sont développées en bioéthique pour limiter a priori, réguler ou contrôler la recherche en amont, c'est-à-dire avant qu'elle ne soit entreprise et qu'elle ne puisse produire des conséquences. Ces tendances s'appuient généralement sur des bases fondamentalistes et intégristes, elles sont radicalement anti-éthiques de l'esprit même de la RDTS. H. Jonas, l'auteur du Principe Responsabilité (1979), célèbre dans le monde de la bioéthique, utilise dans ce contexte une expression remarquable : "l'heuristique de la peur ». Il s'agit d'une curieuse méthode de décision qui enjoint, dans l'incertitude de l'avenir, de prévoir le pire systématiquement, et de décider sur base de l'hypothèse que le pire va se réaliser. Autant dire qu'une telle procédure est très restrictive en ce qui concerne la RDTS. Un argument récurrent dans les discussions bioéthiques est celui de " la pente glissante ou savonneuse »- la slippery slope -. Il revient à affirmer qu'une fois que l'on s'est engagé dans une direction comportant des risques de dérive et d'abus, la pente devient fatale et l'on ne pourra pas empêcher ou s'empêcher d'y succomber. 
27 L'étendue de cette rhétorique est très grande: on la retrouve dans le débat sur l'avortement comme au cœur des objections soulevées contre la recherche en embryologie, en génétique ou la législation de l'euthanasie. Cet argument incarne l'angoisse même de la liberté et de la responsabilité, puisqu'il suppose qu'une fois engagé, on n'est littéralement plus libre ni capable de s'arrêter ou de réajuster un comportement. Cet argument me paraît typique d'un certain profil psychologico-moral de peur à l'égard des pulsions (de l'inconscient) et de refuge dans le surmoi autoritaire et dogmatique imposant des interdits rigoureux et définitifs, absolus, rendant impossible toute perte de contrôle, par empêchement de ce qui pourrait éventuellement y conduire. On condamne ainsi, et par peur anticipée, des entreprises ou des actes innocents voire bénéfiques sous prétexte qu'ils pourraient entraîner des actions mauvaises, en postulant que cette éventualité va se muer en nécessité fatale. L'argument de la pente glissante est caractéristique du doute quant à la capacité de juger compte tenu des circonstances de l'évolution des situations; c'est pourquoi il en appelle à un garde-fou absolu et préventif qui, en interdisant d'une manière massive, épargne l'obligation de juger et de choisir. C'est, je le répète, l'argument antihumaniste par excellence, puisqu'il doute fondamentalement de la faculté humaine de prendre en charge un avenir qui soit évolutif et progressif, apte à tirer les leçons d'une expérience et à avancer d'une façon vigilante et responsable.

\section{Les libertés individuelles}

Lorsque j'ai désigné d'un mot l'enjeu philosophique laïque de la bioéthique, j'ai évoqué la liberté, en précisant que je songeais, d'une part, à la liberté de recherche technoscientifique et, d'autre part, aux libertés individuelles telles qu'elles s'expriment notamment dans la Déclaration universelle des droits de l'homme et dans les constitutions démocratiques. Les techno-sciences biomédicales concernent le corps, la naissance, l'identité, la mort de chaque individu, bref ce qui lui est le plus propre, le plus personnel et privé. Pour une part importante, la bioéthique se voudrait normative : elle veut se constituer en morale et s'exprimer dans des règles de droit énonçant ce que l'individu peut et ne peut pas faire à l'intérieur même de sa sphère la plus privée. Cette volonté procède d'un double postulat :

- les individus seraient généralement incapables de déterminer ce qui est leur propre bien ;

- il y a une morale universelle suffisamment précise pour déterminer les obligations et des interdits concrets et absolus, indépendants des contextes.

29 Dans cette perspective, la bioéthique est un terrain privilégié d'expression des fondamentalismes, des intégrismes et des paternalismes. Ceux-ci n'ont pas le souci d'informer d'une manière neutre et objective, ni d'éduquer à la liberté et à la responsabilité individuelle des choix. Mais la bioéthique est aussi le lieu où ces dernières revendications sont formulées avec force; ainsi en va-t-il du fameux principe d'autonomie et du consentement libre et éclairé (ou volontaire et informé), condition de toute intervention sur une personne, que la bioéthique et la nouvelle éthique médicale n'ont cessé de promouvoir dans leurs déclarations. De même, la bioéthique a encouragé la discussion collective, à des degrés divers, pluraliste ou en tous cas pluridisciplinaire, dans des comités et des commissions d'éthique.

Ce vrai débat bioéthique me paraît être non celui qui oppose entre eux les partisans de morales divergentes, mais celui qui distingue les croyants de quelque conviction qu'ils 
soient, qui pensent disposer de la morale et des vraies valeurs à universaliser éventuellement par la force puisqu'elles sont authentiques, et ceux qui estiment que les questions de valeurs sont irréductiblement plurielles, qu'elles relèvent de la conscience de chacun et qu'il faut seulement veiller à réguler cette multiplicité de la manière la moins injuste possible.

Engelhardt (The Foundations of Bioethics, 1986 et Bioethics and Secular Humanism, 1991) est le philosophe américain de la bioéthique qui a été le plus loin dans cette voie. Il estime que nos sociétés sont irréductiblement polyculturelles, polyethniques, polythéistes, et que la seule morale universelle recevable est formelle, c'est-à-dire qu'elle n'impose aucune conception déterminée et substantielle du bien. Elle énonce seulement que les controverses éthiques doivent être réglées de façon pacifique et qu'on ne peut jamais utiliser la force à l'égard de quelqu'un qui n'en aurait pas lui-même usé. L'État, le droit auraient pour mission essentielle le respect de ce principe et disposeraient d'une force légitime applicable à ceux qui ne l'observeraient pas. Engelhardt exprime ceci en disant que le principe d'autonomie prime le principe de bienfaisance qui implique toujours une conception déterminée du bien. Non pas : "Traite autrui comme tu voudrais être traité ", mais bien : "Traite autrui comme il souhaite être traité », dans la mesure du moins où ce souhait ne heurte pas fondamentalement tes propres valeurs, auquel cas l'abstention est légitime. À noter que cette conception n'exclut pas la discussion ni les moyens pacifiques par lesquels un individu peut essayer de convaincre un autre qu'il a tort et que ce qu'il va faire est, par exemple, dommageable pour sa santé. Mais cette entreprise légitime de persuasion connaît des limites qu'elle ne peut en aucun cas transgresser : le respect de l'autonomie d'autrui quelle que soit la décision qu'il prendra en dernière analyse. Telle est la seule règle éthique universelle applicable entre des personnes conscientes, libres et autonomes. Il s'agit d'un principe de tolérance et de respect mutuel. Chaque individu a le droit et la responsabilité de définir ses valeurs et de s'associer librement avec d'autres individus qui pensent comme lui en des communautés traditionnelles ou nouvelles. Le principe de bienfaisance prend sens et contenu par rapport à celles-ci qui définissent leur conception du bien. Ces conceptions sont différentes et elles déterminent des solidarités diverses. En leur sein, des individus peuvent librement décider de principes communs qui interdiraient, par exemple, l'avortement ou l'euthanasie contraire à leur morale.

\section{Solidarités}

Avec le terme que je viens d'introduire- la solidarité - entre en scène le dernier enjeu que je voudrais souligner. C'est, à mes yeux, l'enjeu le plus difficile à définir d'un point de vue laïque. Pourquoi ? Parce qu'il demande que l'on précise quel serait le contenu du principe de bienfaisance, la conception positive du bien, propre à la communauté laïque. Le laïque a une tendance légitime et compréhensible à souligner surtout la morale formelle, celle de l'autonomie individuelle, de la tolérance universelle. Il sent bien que la communauté dont il fait partie n'est pas tout à fait comme les autres, puisqu'elle place au-dessus de toutes les libertés individuelles, qui sont souvent antithétiques de la solidarité et de l'appartenance communautaire. Le libéralisme et l'individualisme purs ne semblent, par exemple, pas s'opposer à ce qu'un individu vende l'un de ses reins ou sa cornée ou son sang, ou qu'il engage son corps dans des expériences et des manipulations risquées; la tentation existe, sous prétexte de liberté, d'entrer ainsi dans le grand marché, le grand commerce, le grand change et le grand trafic des corps et des produits du corps, après 
celui des biens. Des courants de pensée libertariens sont, à des degrés divers, favorables à ces perspectives auxquelles la bioéthique européenne continentale résiste.

Mon avis à cet égard est nuancé. Idéalement, je ne vois pas au nom de quoi on pourrait légitimement interdire à des individus vraiment libres et informés de faire de leur corps et de leur vie ce que bon leur semble. Reste à définir un individu vraiment libre : tel n'est certainement pas le cas du misérable du Tiers-Monde matériellement enclin à vendre l'un de ses organes; tel n'est pas davantage l'individu peu instruit ou dans une situation quelconque de dépendance psychologique ou sociale ou qui subirait des pressions subtiles de la part de son environnement; tel n'est pas davantage celui qui est hanté par la volonté de pouvoir ou le désir obsessionnel de s'enrichir. La personnalité vraiment autonome est rare et, sans doute, ne fait-elle jamais qu'approcher cet idéal, qui subit tellement les fluctuations du contexte, de l'âge, de la santé. La liberté, au sens de l'autonomie morale, est un idéal que les personnes ne pourraient probablement vraiment réaliser que dans une société idéale qui inclurait tous les êtres humains. Autrement dit, et nous le savons bien, si la laïcité a pour idéal la liberté, elle doit avoir comme communauté morale présomptive la totalité de l'humanité et se soucier d'une solidarité sans bornes. Aussi, lorsque nous militons en faveur de la défense des droits et des libertés individuelles ne devons-nous jamais oublier que ceux-ci n'ont de sens que s'ils s'accompagnent de bien d'autres choses, parmi lesquelles la situation économique et l'éducation, mais aussi l'histoire personnelle de chaque individu. Nous rencontrons ici une objection souvent soulevée, notamment, à propos de l'euthanasie : dans quelles conditions de liberté réelle est-elle demandée ? C'est pourquoi il est si difficile et risqué aussi dans une société qui est loin d'être idéale et parfaitement libre, de promouvoir trop avant ou trop aveuglément les libertés individuelles et d'abandonner entièrement à la sphère privée des décisions dont on sait bien que, dans d'autres circonstances qui, dépendent éventuellement aussi de nous, telle personne ne les aurait pas prises ainsi.

Je ne veux pas, en disant cela, affaiblir mon affirmation selon laquelle, dans le débat bioéthique tel qu'il existe, le rôle fondamental de la laïcité me paraît être celui de la défense des libertés de la personne, je veux seulement dire que ce combat comporte bien des responsabilités et des obligations positives à l'égard des autres. Ces responsabilités peuvent, transitoirement, prendre la forme d'une limitation ou d'un vigilant accompagnement de l'exercice de la liberté qui, sans cela, risque de n'être qu'apparente et, ainsi que je l'ai dit, l'alibi de l'indifférence, de l'égoïsme, du désintérêt ou encore l'instrument du triomphe du plus fort.

Tous les hommes devraient apprendre aujourd'hui à vivre à deux niveaux : celui, d'une part, de leurs convictions morales, identités, appartenances communautaires personnelles et particulières ; celui, d'autre part, de la morale formelle, universelle, qui invite à relativiser le premier niveau et qui consiste dans le respect de l'autonomie d'autrui. C'est le second niveau qui empêche le premier de devenir éventuellement fanatique et dogmatique. Mais la dualité du laïque comporte ceci de spécifique et de difficile : à savoir que sa responsabilité particulière et concrète est la promotion réelle de l'universalité, de la tolérance et de la liberté. La morale laïque du premier niveau a pour contenu la promotion de la morale universelle du second niveau. La communauté laïque est, pour cette raison, à la fois particulière et universelle; elle a et n'a pas une identité propre qui la distinguerait du reste de l'humanité. Là se trouve aussi son ambiguïté, souvent dénoncée, mais inévitable. 


\section{BIBLIOGRAPHIE}

FAGOT-LARGEAULT A., L'homme bio-éthique, Paris, Maloine, 1985.

HOTTOIS G., Le paradigme bioéthique, Bruxelles-Montréal, De Boeck-Erpi, 1990.

HOTTOIS G., et PARIZEAU M.H., coord., Les mots de la bioéthique. Un vocabulaire encyclopédique, Bruxelles-Montréal, De Boeck-Erpi (Paris, Belin diff.), 1993.

LUCAS P., Dire l'éthique. Éthique bio-médicale : le débat, Paris, Actes Sud-INSERM, 1990.

\section{RÉSUMÉS}

Ce que l'on nomme aujourd'hui bioéthique est un excellent révélateur des conditions dans lesquelles la question éthique se pose en général dans les sociétés développées complexes modernes. Définir une éthique adaptée aux caractères 2 spécifiques de ces sociétés implique de distinguer deux formes d'obligations éthiques: celle des règles dogmatiques découlant de l'appartenance à des communautés particulières, et celle d'une morale formelle en laquelle s'indique l'idéal d'une communauté laïque dont l'ambiguïté inévitable est d'être à la fois particulière et universelle.

INDEX

Mots-clés : conscience morale, éthique, liberté, système de valeurs, bioéthique

\section{AUTEUR}

\section{GILBERT HOTTOIS}

Professeur en philosophie, Université libre de Bruxelles, directeur du Centre de recherches interdisciplinaires en bioéthique (CRIB), Belgique 